\title{
Talk about the Quality Control of Steel Sleeve Extrusion Connection Technology
}

\author{
Urban-rural Architectural Institute, Minxi Vocational and Technical College.Fujian Longyan 364021
}

\section{Introduction}

With the development of the construction industry, high-rise buildings, large span and special structure are increasing, the application of building reinforcement is developed in large diameter, dense layout and high strength, It is difficult to meet the needs of traditional rebar connection technology, such as lap binding, bonding arc welding, flash butt welding and gas pressure welding. In recent years, many large engineering projects have used sleeve extrusion and taper thread connection technology. Extrusion sleeve connection is to put the two waiting the end of the bar to insert a high-quality steel sleeve, and then used extruder in the number of lateral pressure, casing tightly bite after plastic deformation and ribbed bar to achieve the objective of the connection. The advantages of sleeve extrusion connection are high strength and reliable quality; safe operation, no open fire, not affected by climate; connection strong adaptability and can be used for vertical, horizontal, oblique, altitude, underwater and all other steel bar connection, is particularly applicable to certain chemical composition be not suitable for the traditional welding process of steel connections, such as special steel, steel imports. It is mainly used for the connection of the diameter of 20 $\sim 40 \mathrm{~mm}$ with rib bar. ${ }^{1}$

This paper analyzes the quality control of the steel sleeve extruding technology in the construction engineering, so as to facilitate the better application of the technology in the future construction.

\section{Quality control and prevention measures}

1.1 The two longitudinal ribs are not in the same plane

It is mainly due to the pressing mode movement direction be not perpendicular to the plane of the two longitudinal ribs. There are many reasons, such as before the reinforcement be not connected to the same plane; when the material is turned off, the longitudinal ribs are not calculated.

Prevention and control measures: According to the position of the sleeve indentation position, the position of the positive pressure die and the movement direction of the pressing die are perpendicular to the plane of the longitudinal rib of the steel bar, which is to ensure that the maximum contact surface is on the transverse rib of the steel bar. $^{2}$

\subsection{Eccentric bend}

Eccentricity and bending are shown in: the axis of the connected reinforcement is not on the same axis as the axis of the sleeve, and the head bend is greater than $4^{\circ}$.

Mainly due to:

(1) Not cut or straighten the steel bar.

(2) The steel bars are not squared when pressed.

\begin{abstract}
This article introduced the advantages of steel sleeve extrusion connection technology, the quality defects and prevention measures of ribbed reinforced steel sleeve in the process of construction, put forward the main measures of steel sleeve extrusion connection technology in the construction.
\end{abstract}

Key words: cold extrusion; connection; quality

Published online: 30th Sept, 2017

Prevention and control measures:

(1) Remove or straighten the steel bend.

(2) To put the steel bar on the same axis as the steel bar; adjust the pressure clamp to control the indentation mark on the surface of the sleeve, and make the pressing of the pressure die perpendicular to the steel sleeve axis; in the process of reinforcement, always pay attention to the alignment of the two ends of the joint. ${ }^{3}$

1.3 Air pressure and indentation are not evenly distributed 
The uneven distribution of pressure and indentation is shown in: The length of the steel sleeve is not enough; the indentation is obviously uneven.

Mainly due to:

(1) Do not check the length of the steel bar to the sleeve.

(2) Do not press the steel bar into position sign extrusion.

(3) No indentation marks on the sleeve, or pressure die and check marks are not right.

Prevention and control measures:

(1) Before construction, position mark and check mark on steel bar. The distance of the locating marker is half of the length of the sleeve, and the distance between the check mark and the positioning mark is: When the length of steel sleeve is smaller than $200 \mathrm{~mm}$, take $10 \mathrm{~mm}$; when the length of the steel sleeve is greater than $200 \mathrm{~mm}$, take $15 \mathrm{~mm}$.

(2) Press the pressure mark on the sleeve to squeeze, when extruding, the pressure of the clamp should be aligned to the sleeve indentation mark, and perpendicular to the axial compression steel axis, the extrusion should be pressed from the center of the sleeve to the end. ${ }^{4}$

2.4 The steel bar can't go into the supporting sleeve

Mainly due to:

(1) Small diameter of sleeve.

(2) The inner diameter of the sleeve is reduced.

(3) The end of the rebar has a horseshoe, bending or oversize.

Prevention and control measures:

(1) The steel bar is twisted, bent should be cut or straightened, the end of the side of the longitudinal rib is too large, it should be used in the grinding wheel, or the flat rib bar, which is forbidden to be cut with electric welding. The cutting surface of the steel bar should be perpendicular to the steel axis.

(2) Rebar approach with vernier caliper check, large steel bar is for return handling.

(3) The sleeve shall have a factory certificate. The sleeve shall be stacked in good order according to different specifications in transportation and storage, and shall not be stacked in the open air to prevent rust and contamination. ${ }^{5}$

\section{Main measures taken in construction}

3.1 Fixed operation workers, and technical training for operators, certified and certified.

3.2 Before the extrusion con-nection, the rebar is marked with the positioning mark and check mark.

3.3 Pressure marks must start from the middle of the joint to the two ends. ${ }^{6}$

3.4 The cold extrusion sleeve shall be in accordance with the specifications of the steel bar, and shall not be small in the large generation. When the reinforce-ment is in diameter, a large diameter sleeve can be used if the steel bar is different; if there are two levels of reinforcement, a transition must be added. ${ }^{7}$

3.5 The pressure die of cold extrusion must be used for the specification. When the steel reinforcement with different diameter is connected, the corresponding should be adopted according to the steel diameter of the two sides.

3.6 In order to improve the efficiency of the work, the steel sleeve is connected with the steel bar in the processing field, and the half of the extrusion head is finished, and the other half is extruded on the spot, but the semi-finished product should be placed in place to prevent the extrusion cylinder from being polluted.

3.7 After the extrusion connection is completed, it should be straightened in time, and the angle of deflection is not more than $4^{\circ} .8$

3.8 To strengthen quality inspection work, quality inspection is divided into appearance inspection and tensile test.

(1) All the joints should be inspected by the construction team, and the professional quality inspectors shall check $10 \%$ of the joint.
(2) In the tensile test, 500 joints with the same batch number steel sleeve and the same production condition are one batch, and less than 500 joints still serve as a piece. Three joints are drawn in each batch. ${ }^{9}$

3.9 Cold extrusion equipment should be serviced once every 6,000 uses. The high-pressure rubber tube in the extruded equipment is vulnerable. It should be prevented from dragging, bending, or being carved by steel bars. ${ }^{10}$

\section{Conclusion}

At present, the steel sleeve extrusion technology is widely used in construction engineering, with high joint strength and good quality stability. For this technology, the rougher and more economical of the connected steel bars, considering the economic factors and the convenience of construction, when the diameter of reinforcement is greater than 22, it is reasonable. The cold extrusion connection is used to solve and relieve the reinforce-ment of the reinforcing steel bar in the beam column, and the contradiction of the placement is favorable to the construction of concrete. Specification requires that the diameter of $\phi 22$ or unfavorable tie bar connection, and can not be used in seismic requirements engineering, con-nected by cold extrusion, can meet the specification requirements, ensure the safety and seismic performance of the structure, has great social benefits.

\section{References}

[1] Jin Lan. Introduction to extrusion of rib cage with rib cage [J]. South steel technology and management, 2011,03: 62-63.

[2] Zheng Huaiming, Chang Hongmin. The quality control of the joint of the rib cage [J]. Shanxi Building, 2003,29 (13): 87-88.

[3] Lei Shanjun, Geng Ping, Hu Hao. The construction technique of cold extrusion of rib bar [J]. Henan Water conservancy, 2003,(5): 20. 
[4] Chen Qiang, Zhuang Qingjin. Application of cold extrusion connection technology for rib cage with rib cage [J]. Chinese municipal engineering, 2006,(1): 60+63+92.

[5] Pan Zhenyong. Application of reinforced cold extrusion sleeve connecting technology in engineering $[\mathrm{J}]$. Science and technology information, 2008,(16): 72.

[6] Li Hongwei. Application of steel sleeve extruding connection technology [J]. Chinese roads, Cryogenic construction technology, 2005,(13): 48. 2004,(2):28.

[7] Li Fugui, Fu Zhiqin, Guo Xiufang, Zhang Laiwen. Technology and its examples of rib cage extruding with rib cage [J]. Heilongjiang Water conservancy technology, 2005,(5): 31.

[8] Zhang Yongliang. The extrusion process of large diameter steel bar sleeve is used in engineering [J].
[9] Sun Hanan, Yuan Feng, Li Yetao. The technology and application of rib bar with rib bar [J]. Heilongjiang transportation technology, 2002,(7):19.

[10] Ma Quan'an, Zhu Lijun. Application of rib cage extruding connection technology [J]. Shandong Building materials, 2001,(4):30. 